\title{
Which physical signs help most in the diagnosis of leprosy? A proposal based on experience in the AMFES project, ALERT, Ethiopia
}

\author{
PAUL SAUNDERSON \& GUIDO GROENEN \\ ALERT, PO Box 165, Addis Ababa, Ethiopia
}

Accepted for publication 10 January 2000

Summary As integration of leprosy control programmes proceeds, general health staff will have responsibility for the diagnosis of most new cases of leprosy. The training required by these workers has not yet been set out in detail. In this paper the criteria for making the diagnosis of leprosy in the AMFES cohort of 594 new cases are examined. Since this study does not include details of suspects in whom leprosy was excluded on clinical grounds, true sensitivity and specificity values cannot be calculated, but the positive predictive value of the diagnostic criteria can be measured. Sensory loss in a typical skin patch is the most important sign of early leprosy, but was not present in 132 (49\%) of the 268 cases with a positive skin smear. Thickening of the ulnar nerve is a valuable sign of leprosy in Ethiopia. It can be taught to health workers, who can practise by examining their own ulnar nerves. It is more likely to be present than nerve function impairment and is particularly important when skin smears are difficult to do or are unreliable. We recommend that five basic signs are used, the presence of any two being diagnostic of leprosy:

- Skin lesion(s) consistent with leprosy.

- Loss of sensation in such a lesion.

- Thickening of either ulnar nerve.

- Loss of sensation in the palm of the hand or the sole of the foot.

- The presence of acid-fast bacilli in skin smears.

Exact policies for the diagnosis of leprosy should be worked out and validated for each national programme.

\section{Introduction}

Leprosy is becoming less common in most previously endemic areas. Most traditional vertical leprosy control programmes are handing over their work to integrated or combined programmes, in which routine patient management is carried out by general health staff in a peripheral clinic. ${ }^{1,2}$ There are many potential advantages of this change, not least of which is increased cost-effectiveness.

\section{Correspondence to: P. Saunderson}


The major disadvantage anticipated, however, is the lack of specialized skills in these general health workers, who have to manage a variety of conditions and for whom leprosy will usually be a rare problem. Those involved in the training of health staff have expressed concern about defining a basic curriculum related to leprosy, which would not overload the trainee, but which at the same time would provide a sound basis for the management of the cases being seen. ${ }^{3-5}$

This paper looks at one aspect of a basic curriculum, namely the diagnosis of leprosy and what would be the simplest message to teach general health workers. The objective of such training would be to enable them to suspect leprosy and either make the diagnosis themselves in a reliable manner, or refer the case to someone with more experience.

Traditionally, three cardinal signs have been used to diagnose leprosy, ${ }^{6}$ namely:

1. Definite loss of light touch sensation in a skin lesion consistent with leprosy.

2. Skin smears positive for acid-fast bacilli.

3. Thickening of one or more peripheral nerves.

These signs have not been extensively tested for sensitivity, specificity and interobserver agreement, and studies that have been published give equivocal results. ${ }^{7-9}$

Current WHO guidelines, however, advocate the use of only two cardinal signs, numbers 1 and 2 above, ${ }^{10}$ either one being sufficient to make the diagnosis of leprosy. These guidelines further state that: 'nerve damage, mainly to peripheral nerve trunks, constitutes another feature of leprosy. There may be loss of sensation in the skin and weakness of muscles supplied by the affected nerve. In the absence of these signs, nerve thickening by itself, without sensory loss and/or muscle weakness is often not a reliable sign of leprosy.'

The role of nerve thickening in the diagnosis of leprosy is made clear in the seventh report of the WHO Expert Committee on Leprosy, ${ }^{11}$ which stated that 'the disease should not be diagnosed if only nerve thickening is present, without any other accompanying symptoms or signs.'

We decided to examine the criteria for the diagnosis of leprosy in a large prospective study being carried out in Ethiopia, in order to determine what may be the most appropriate diagnostic criteria for use by peripheral health staff.

\section{Patients and methods}

Unselected new cases were enrolled in the AMFES project (ALERT MDT Field Evaluation Study) between March 1988 and March $1993 .{ }^{12}$ For a variety of reasons related to deeply held cultural beliefs and practices, patients with leprosy in central Ethiopia have generally presented very late for diagnosis; signs of nerve involvement are therefore rather common in this group. ${ }^{13}$ Detailed records of the clinical signs at diagnosis were available for analysis.

The following details for each case were analysed:

- Skin smear result.

- The number of skin lesions.

- The presence of loss of sensation in a skin lesion, tested with a wisp of cotton wool.

- The presence of nerve function impairment, based on the testing of four muscles for power, 
using a three-point scale of strong/weak/paralysed (eye closure, fifth finger abduction, thumb abduction and dorsiflexion of the foot) and 10 points on each palm and sole for loss of touch sensibility, using a $10 \mathrm{~g}$ nylon monofilament. Details of any loss of muscle power and any loss of touch sensibility were recorded.

- The presence of visible damage to hands or feet (who impairment grade 2). ${ }^{14}$

- The presence of thickening in any of six nerves examined (greater auricular, ulnar, median, radial cutaneous, peroneal and posterior tibial).

- Skin biopsy results for certain patients.

\section{Results}

In all, 603 new cases were enrolled in AMFES. Nine patients were excluded on the grounds of incorrect diagnosis (three cases) and incorrect enrolment procedures (6 cases), leaving 594 cases for the current analysis.

Biopsies were performed for 10 cases in whom the diagnosis on clinical grounds (usually typical skin lesions without sensory loss) was suggestive but not certain. Leprosy was confirmed in nine cases (90\%). Biopsies were also done on a random sample of 102 patients whose diagnosis was not considered doubtful by experienced field staff. Leprosy was confirmed in 100 of these cases $(98 \%)$.

Figure 1 shows how the traditional cardinal signs of leprosy contributed to the diagnosis in 594 AMFES patients. In this analysis, thickening in any of six nerves was taken into

\section{Thickened \\ nerves: \\ $496(84 \%)$}

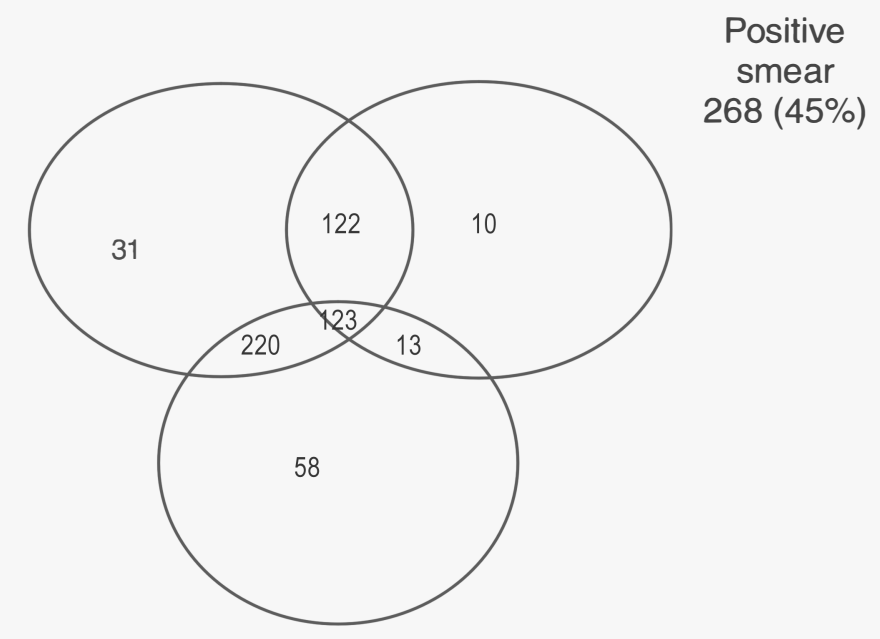

Sensory
loss in skin
lesions
$414(70 \%)$

Figure 1. Traditional criteria for the diagnosis of leprosy (AMFES). $n=594 ; 17$ cases are undiagnosed using these criteria. 
Table 1. The contribution of different nerves to the diagnosis of leprosy

\begin{tabular}{lccr}
\hline Cases with any nerve thickening & $\begin{array}{c}\text { Cases with ulnar } \\
\text { nerve thickening }\end{array}$ & $\begin{array}{c}\text { Cases with radial } \\
\text { cutaneous nerve } \\
\text { thickening }\end{array}$ & $\begin{array}{c}\text { Cases with either } \\
\text { ulnar or radial } \\
\text { cutaneous thickening }\end{array}$ \\
\hline 496 cases in total & $\begin{array}{r}403(81 \%) \\
25(81 \%)\end{array}$ & $\begin{array}{r}283(57 \%) \\
19(61 \%)\end{array}$ & $\begin{array}{r}443(89 \%) \\
31 \text { cases without other diagnostic signs }\end{array}$ \\
\hline
\end{tabular}

account, all positive smears and any sensory loss in skin lesions. Seventeen cases did not show any of the three cardinal signs: they were diagnosed as follows:

Clinical suspicion confirmed by biopsy

Strong clinical suspicion alone (including one child)

when a biopsy could not be done for logistic reasons

Nerve function impairment, without a cardinal sign
9 cases

6 cases

2 cases

The ulnar and radial cutaneous nerves were the most commonly involved, as shown in Table 1 . The one case diagnosed by the finding of a thickened nerve, but without thickening of either the ulnar or radial cutaneous nerves, had a grossly thickened median nerve and some loss of function of the ulnar nerve.

Because of the very frequent involvement of the ulnar nerve and the relative ease with which it can be examined, Figure 2 looks at the effect of using only that nerve for diagnostic purposes.

Figure 3 shows how the new WHO guidelines would work in practice in this cohort of patients in Ethiopia, using positive smears, sensory loss in the lesions and nerve function impairment as the three cardinal signs. Of the 310 patients with impairment, 277 (89\%)

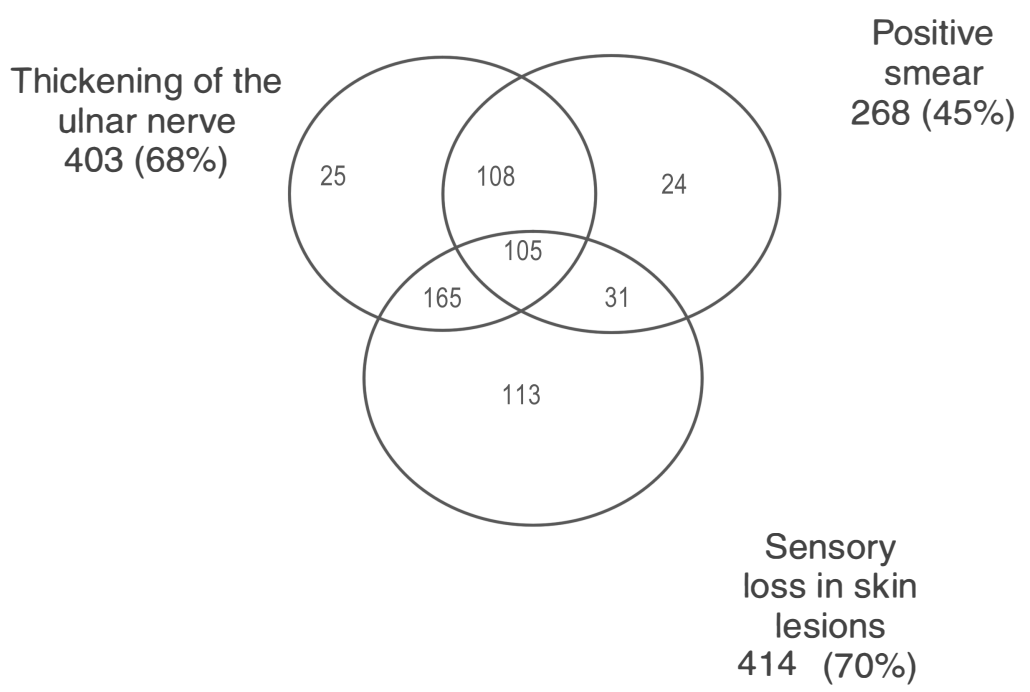

Figure 2. Using the traditional cardinal signs, but restricting the examination of nerves to the ulnar alone (AMFES). $n=594 ; 23$ cases are undiagnosed using these criteria. 


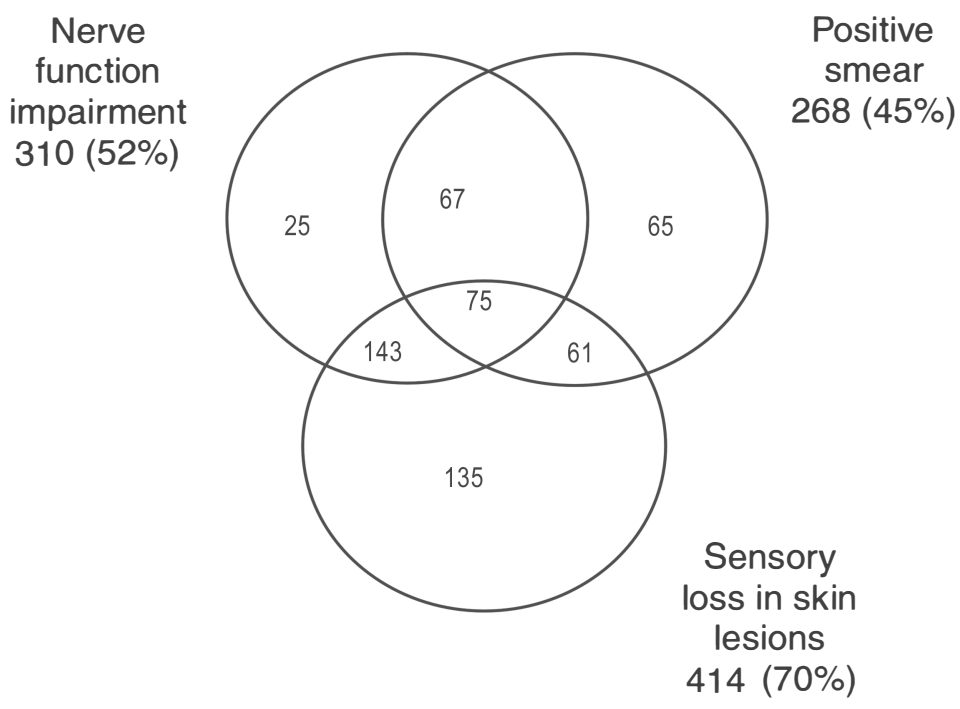

Figure 3. WHO criteria for the diagnosis of leprosy (AMFES). $n=594 ; 23$ cases are undiagnosed using these criteria.

had sensory loss, while only 160 (52\%) had loss of muscle strength. By examining sensory loss and loss of muscle strength as two different criteria, it is found that sensory loss is a much more sensitive diagnostic tool; if both tests are used, loss of muscle strength contributes very little, with only one additional case being diagnosed.

The simplest group of signs with the maximum yield in this patient population consists of four criteria, as shown in Figure 4, with any one sign being strongly suggestive of leprosy:

- A skin smear positive for acid-fast bacilli.

- Sensory loss in a typical skin lesion.

- Loss of sensation anywhere in the palms of the hands or soles of the feet.

- Thickening of one or both ulnar nerves.

The most worthwhile addition to this battery of tests would be to add thickening of the radial cutaneous nerve: three additional cases would be diagnosed, bringing down the number of undiagnosed cases to 14 . Voluntary muscle testing and an assessment of damage to the hands and feet (WHO impairment grade 2) would add very little to the diagnostic process, even in this cohort with relatively high rates of impairment at diagnosis.

\section{Discussion}

When proposing diagnostic criteria for any medical condition, the sensitivity, specificity and predictive values of the criteria are important considerations. Table 2 shows how these terms are defined and calculated. In leprosy, a test with low sensitivity will mean that new cases are missed, with the possible outcome of permanent nerve damage, disability and deformity. Low specificity, on the other hand, will lead to overdiagnosis and the unnecessary labelling 


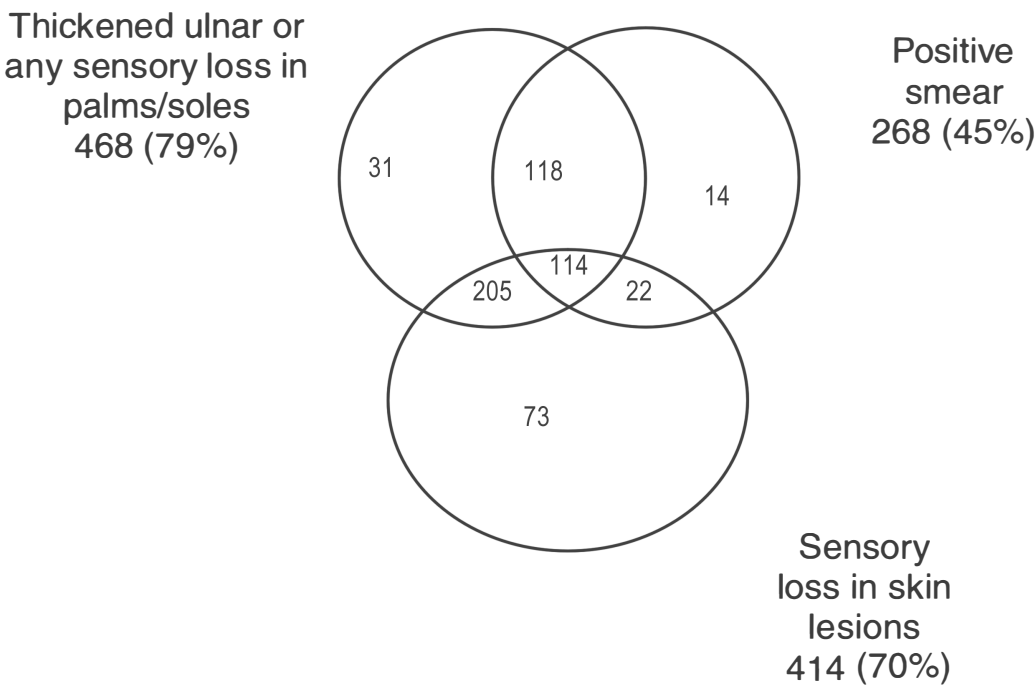

Figure 4. The use of four basic signs for the diagnosis of leprosy (AMFES). $n=594 ; 17$ cases are undiagnosed using these criteria.

of some people as leprosy patients, still associated with varying degrees of stigma in many cultures.

For a rare condition, such as leprosy now is in most countries, the specificity of a diagnostic test must be very close to $100 \%$. If the specificity is much less than this, there will be many false positives and a large proportion of the patients diagnosed would not actually have the disease: in other words the positive predictive value of the test would be very low. In practice, the positive predictive value of diagnostic tests in this situation is improved by pre-selection of cases: ${ }^{15}$ for leprosy, clinic attenders with suggestive skin lesions are

Table 2. The definition and calculation of terms relating to diagnostic tests

\begin{tabular}{llll}
\hline & \multicolumn{3}{c}{ True disease status } \\
\cline { 2 - 4 } Test result & Present & Absent & Total \\
\hline Positive & A & B & A + b \\
Negative & C & D & C + d \\
Total & $\mathrm{a}+\mathrm{c}$ & $\mathrm{b}+\mathrm{d}$ & $\mathrm{a}+\mathrm{b}+\mathrm{c}+\mathrm{d}$ \\
\hline
\end{tabular}

Definitions: $a=$ true positives; $b=$ false positives; $c=$ false negative; $d=$ true negatives.

Sensitivity $=a /(a+c)$ or the proportion of all those who really have the disease who come out positive in the test.

Specificity $=d /(b+d)$ or the proportion of all those who do not have the disease who come out negative in the test.

Positive predictive value $=a /(a+b)$ or the proportion of those with a positive test who really have the disease. 
pre-selected to undergo the more formal diagnostic tests and the prevalence of leprosy will be much higher in this group than in the general population.

The specificity of a diagnostic test can only be measured when a large number of suspects are examined and the proportion of false positives is calculated. The gold standard for determining the actual status of each subject (leprosy: yes or no) is difficult to define, but is likely to include a combination of expert clinical opinion and histopathological examination. ${ }^{7}$ As the present study neither took biopsies nor recorded details of suspects who were examined and thought not to have leprosy, it is impossible to calculate the specificity of this battery of diagnostic tests from this dataset. However, in those cases biopsied as a random check of the accuracy of the clinical diagnosis, only $2 \%$ were found to be falsely positive, indicating a positive predictive value of $98 \%$ in this setting.

In this cohort, cases missed by the various diagnostic regimens described, range from 14 to 23 out of a total of 594 new cases. This difference may well be smaller than the errors to be expected in any routine programme. The field staff in the AMFES project were very experienced and worked in a vertical programme, so that their performance may not be reproducible in a general clinic. On these grounds, therefore, any of the methodologies described would be acceptable in terms of sensitivity.

The skin smear is the only part of the diagnostic process which is outside the direct control of the primary healthcare worker. Given appropriate training and motivation, (s)he can carry out all the other diagnostic steps. In many programmes, therefore, it is not surprising that the skin smear is the weak link and many leprosy suspects may wait a long time for a smear result, or never have the possibility of a skin smear at all.

Using the traditional diagnostic criteria (Figure 1), there are only 10 patients diagnosed solely by a positive smear, while 478 (80\%) of patients are diagnosed by at least two criteria. If the number of nerves examined is reduced to one (Table 1, Figure 2), the figures are not greatly changed. Using the new WHO guidelines (Figure 3), 65 patients are diagnosed by smear alone, while only $346(58 \%)$ are diagnosed by at least two criteria. The addition of ulnar nerve thickening as a diagnostic sign (Figure 4) greatly improves the possibility that two signs are available to suggest the diagnosis of leprosy. This could be an important consideration in areas where smears are not available.

Similar data have been published from Bangladesh in a study which examined the classification of new cases. ${ }^{16,17}$ A total of 244 consecutive new cases were diagnosed clinically, with skin smears and biopsy. It was found that $32 \%$ had positive skin smears, $77 \%$ had loss of sensation in a skin lesion, 90\% had enlarged nerves and 54\% had nerve function impairment; of the 32 cases not confirmed by biopsy or skin smears, 24 had loss of sensation in a skin lesion, seven had enlarged nerves and one had nerve function impairment. No combined figures are available, since data were collected for classification purposes, not diagnosis, but the similarity with the AMFES data as illustrated in Figures 1 and 3, is striking.

The reliability of thickened nerves as a sign of leprosy was first questioned in Nepali army recruits, $63 \%$ of whom had thickened greater auricular nerves. ${ }^{18}$ It was suggested that well-developed musculature could lead to enlargement of the related nerve, an effect noted in the ulnar nerves of another group of manual workers. ${ }^{19}$ However, in a detailed clinical and histological study, Srinivas et al. found that in a carefully selected group of suspects with nerve enlargement but no other clinical sign of leprosy, five of $16(31 \%)$ had leprosy, two had diabetic neuropathy and nine were labelled as idiopathic neuropathy. ${ }^{20}$ 
In a large population survey in Malawi, the sensitivity and specificity of the cardinal signs of leprosy were examined: $95 \%$ of the cases diagnosed were early paucibacillary cases. ${ }^{7}$ It was noted that amongst self-reporting new cases in Malawi, 49.5\% had definite nerve enlargement, while the figure was only $19.2 \%$ for actively detected cases. Definite nerve enlargement was said to make the diagnosis of leprosy 'extremely likely', although it contributed to the diagnosis in only a minority of cases of paucibacillary disease. It is interesting that in the same survey, sensory loss in skin lesions was found unsatisfactory as a diagnostic test with a sensitivity of $48.5 \%$ and a specificity of $72 \%$. The conclusion of the Malawi study was that the diagnosis of very early leprosy is so uncertain by clinical methods alone that such a total population survey should not be undertaken without the help of histopathology.

In the cohort presented here, nerve enlargement is shown to be a valuable sign in skinsmear positive cases. These patients may have poorly defined skin lesions, which often do not show sensory loss. All four figures show that 132 (49\%) of skin-smear positive cases do not display the cardinal sign 'definite loss of light touch sensation in a skin lesion consistent with leprosy'. If skin smears are not easily available, Figures 3 and 4 indicate that ulnar nerve palpation could greatly increase the sensitivity of the diagnostic procedure for this very important group of patients.

The most recent report of the WHO Expert Committee on Leprosy defines a case of leprosy as a person having one or more of the following features: ${ }^{11}$

1. Hypopigmented or reddish skin lesion(s) with definite loss of sensation.

2. Involvement of the peripheral nerves, as demonstrated by definite thickening with loss of sensation.

3. Skin smears positive for acid-fast bacilli.

The first two features are, in fact, double signs. We propose splitting them into their component parts, making five signs of leprosy, any two of which would be grounds to diagnose the disease. This means that a typical case with early lepromatous leprosy, who has skin lesions without definite sensory loss and thickened nerves without impairment, could be diagnosed even if a skin smear result is not available. While a positive skin smear on its own should be enough to diagnose leprosy, it is unlikely to be found without any of the other four signs.

We therefore recommend that those general health staff who are to be responsible for the diagnosis of leprosy should be taught to look for five signs:

1. Skin lesion(s) consistent with leprosy.

2. Loss of sensation in such a lesion.

3. Thickening of either ulnar nerve.

4. Loss of sensation in the palm of the hand or the sole of the foot.

5. The presence of acid-fast bacilli in skin smears.

Finding any two of these signs would be diagnostic of leprosy.

The exact policy for diagnosis must be determined by each national programme; it may be necessary to recommend that certain suspects are referred to a specialist for confirmation of the diagnosis. Further research into the sensitivity and specificity of these diagnostic criteria in different endemic countries is needed and the best methods for teaching general health staff remain to be elucidated. 


\section{Acknowledgements}

We thank Dr Shibru Gebre and the staff of the ALERT Leprosy/TB Control Division for their dedication and perseverance in managing the patients and collecting data over so many years. The financial support of ILEP, through Netherlands Leprosy Relief (NLR), has been constant throughout the study and is gratefully acknowledged. We thank ALERT as a whole for institutional and administrative support. We also thank the anonymous reviewers of this paper, who gave very helpful comments on the draft manuscript.

\section{References}

${ }^{1}$ Feenstra P. Leprosy control through general health services and/or combined programmes. Lepr Rev, 1993; 64: 89-96.

2 Saunderson PR. Joint Tuberculosis/Leprosy Programmes. Lepr Rev, 1997; 68: 2-9.

3 McDougall AC. Training in leprosy: does the current strategy need revision? Lepr Rev, 1995; 66: 89-95.

${ }^{4}$ Groenen G, Alldred N, Nash J. Comment: training in leprosy: the training needs for Africa, and the role of large training institutions. Lepr Rev, 1996; 67: 148-150.

5 Saunderson PR. Future role of leprosy training and/or research institutions. Lepr Rev, 1998; 69: 160-163.

${ }^{6}$ Hastings RC. Leprosy, 1st edn. Churchill Livingstone, New York, 1985.

7 Ponnighaus J M, Fine PEM. Leprosy in Malawi: 1. Sensitivity and specificity of the diagnosis and the search for risk factors for leprosy. Trans R Soc Trop Med Hyg, 1988; 82: 803-809.

${ }^{8}$ Sirumban P, Kumar A, Durai V, Neelan PN. Diagnostic value of cardinal signs/symptoms in paucibacillary leprosy. Ind J Lepr, 1988; 60: 207-214.

9 Gupte MD, Vallishayee RS, Nagara ju B, Ramalingam A, Lourdusamy G, Kannan S. Inter-observer agreement and clinical diagnosis of leprosy for prophylaxis studies. Indian J Lepr, 1990; 62: 281-295.

10 WHO. A Guide to Eliminating Leprosy as a Public Health Problem. WHO, Geneva, 1995.

11 WHO Expert Committee on Leprosy. Seventh Report. Technical Report Series 874; 1998.

12 de Rijk AJ, Gebre S, Byass P, Berhanu T. Field evaluation of WHO-MDT of fixed duration at ALERT, Ethiopia: the AMFES project - I. MDT course completion, case-holding and another score for disability grading. Le pr Rev, 1994; 65: 305-319.

13 Bekri W, Gebre S, Mengiste A, Saunderson PR, Zewge S. Delay in presentation and start of treatment in leprosy patients: a case-control study of disabled and non-disabled patients in three different settings in Ethiopia. Int $J$ Lepr, 1998; 66: 1-9.

14 WHO Expert Committee on Leprosy. Sixth Report. Technical Report Series 768; 1988.

15 Toman K. Sensitivity, specificity and predictive value of diagnostic tests. Bull Int Union Tuberc, 1981; 56: 18-27.

16 Groenen G, Saha NG, Rashid MA, Hamid MA, Pattyn SR. Classification of leprosy cases under field conditions in Bangladesh. I. Usefulness of skin-smear examinations. Lepr Rev, 1995; 66: 126-133.

17 Groenen G, Saha NG, Rashid MA, Hamid MA, Pattyn SR. Classification of leprosy cases under field conditions in Bangladesh. II. Reliability of clinical criteria. Lepr Rev, 1995; 66: 134-143.

18 Lynch P. Greater auricular nerve in the diagnosis of leprosy. BMJ, 1978; 1340.

19 Dharmendra. Thickened nerves in diagnosis of leprosy. Lepr Ind, 1980; 52: 1-2.

20 Srinivas HV, Lakhani R, Mehta LN, Antia NH. Study of thickened nerves in a leprosy endemic region. Lepr Ind, 1980; 52: 53-64. 